

\title{
Some properties of point processes in statistical optics
}

Bernard Picinbono, Cherif Bendjaballah

\section{To cite this version:}

Bernard Picinbono, Cherif Bendjaballah. Some properties of point processes in statistical optics. Physical Review A: Atomic, molecular, and optical physics [1990-2015], 2010, 82 (3), pp.638111638118. 10.1103/PhysRevA.82.063811 . hal-00547053

\section{HAL Id: hal-00547053 https://hal.science/hal-00547053}

Submitted on 15 Dec 2010

HAL is a multi-disciplinary open access archive for the deposit and dissemination of scientific research documents, whether they are published or not. The documents may come from teaching and research institutions in France or abroad, or from public or private research centers.
L'archive ouverte pluridisciplinaire HAL, est destinée au dépôt et à la diffusion de documents scientifiques de niveau recherche, publiés ou non, émanant des établissements d'enseignement et de recherche français ou étrangers, des laboratoires publics ou privés. 


\title{
Some properties of point processes in statistical optics
}

\author{
B. Picinbono* and C. Bendjaballah ${ }^{\dagger}$ \\ Laboratoire des Signaux et Systèmes, Centre National de la Recherche Scientifique, and \\ École Supérieure d'Électricité, 3 rue Joliot-Curie, F-91192 Gif-sur-Yvette, France
}

(Received 31 March 2010; published 8 December 2010)

\begin{abstract}
The analysis of the statistical properties of the point process (PP) of photon detection times can be used to determine whether or not an optical field is classical, in the sense that its statistical description does not require the methods of quantum optics. This determination is, however, more difficult than ordinarily admitted and the first aim of this paper is to illustrate this point by using some results of the PP theory. For example, it is well known that the analysis of the photodetection of classical fields exhibits the so-called bunching effect. But this property alone cannot be used to decide the nature of a given optical field. Indeed, we have presented examples of point processes for which a bunching effect appears and yet they cannot be obtained from a classical field. These examples are illustrated by computer simulations. Similarly, it is often admitted that for fields with very low light intensity the bunching or antibunching can be described by using the statistical properties of the distance between successive events of the point process, which simplifies the experimental procedure. We have shown that, while this property is valid for classical PPs, it has no reason to be true for nonclassical PPs, and we have presented some examples of this situation also illustrated by computer simulations.
\end{abstract}

DOI: 10.1103/PhysRevA.82.063811

PACS number(s): 42.50.Ar, 05.40.-a, 02.50.-r, 02.70.Rr

\section{INTRODUCTION}

In a previous paper [1] it was shown that the analysis of the statistical properties of the point process (PP) of photon detection times allows us to determine whether or not an optical field is classical, in the sense that its statistical description does not require the methods of quantum optics. For this purpose the basic idea was the fact that the PP associated with a classical field is necessarily a doubly stochastic (DS) Poisson process [2], which means a Poisson process with a random density, and introduces various specific properties. If at least one of these properties is then violated, the corresponding optical field is certainly nonclassical. Among these properties one of the best known and used in various experimental results is the so-called antibunching effect [3-7].

Such an effect cannot appear with a DS Poisson process. Yet this reasoning immediately suggests the converse question: Is an optical field that exhibits the bunching effect necessarily classical? In order to answer this question it is necessary to study more carefully the properties of PPs that can be used for the description of photon detection. For example, the previous question leads to the search for a model of PP which is not a DS Poisson but such that its coincidence function as defined in [1] exhibits a bunching effect. If such a model exists, regardless of the question of its physical realization, we can conclude that the bunching effect is not a characteristic property of classical fields. The same reasoning can be presented for various other properties appearing with nonclassical fields and this leads to the main purpose of this paper which is to investigate some models of PPs that can be used to analyze various examples of situations appearing in quantum optics.

It is important to note that the statistical properties of a PP appearing in photon detection can, at least theoretically and by using standard methods of quantum optics, be deduced from

\footnotetext{
*Bernard.Picinbono@1ss.supelec.fr

${ }^{\dagger}$ Cherif.Bendjaballah@1ss.supelec.fr
}

the density matrix $\rho$ describing completely the statistics of the optical field. The inverse problem is much more complicated and it is often an open question to know whether or not it is possible to associate with a given mathematical PP a density matrix $\rho$ such that this PP describes the photon detection phenomenon in the optical field defined by $\rho$. If this is possible we shall say that the PP considered is a detection PP (DPP). Since we cannot ensure that the PPs used in this paper are DPPs, we shall use for our discussion the models of PPs the description of which is the simplest possible. This leads immediately to renewal processes.

They are characterized by the fact that the intervals between successive points, sometimes called lifetimes of order one, are independent and identically distributed (IID) positive random variables. Such PPs are then completely defined by the probability distribution of these random variables. If this class of models is too restrictive for our purpose, it is possible to introduce some correlations between successive intervals of points and a preliminary example in this direction was introduced in [1].

The paper is organized as follows. In Sec. II we review the principal notions of PPs concerning their use in statistical optics. The notations are in general the same as in [1] in order to make our review as short as possible. In particular, as the objective is the comparison between classical and nonclassical optical fields by using statistical properties of photoelectron PPs, we introduce a set of three properties of these PPs that must be satisfied when the field is classical. These properties correspond to the three kinds of experiments usually presented in the literature concerning the statistical analysis of optical fields, namely the bunching effect, the time intervals between successive occurrence points and the photon counting. If at least one of these properties is not verified the field is not classical. These properties can be expressed in terms of coincidence function, of the probability density function (PDF) of the lifetime and of photon counting. Section III is devoted to the review of main properties of renewal PPs that can be used in the analysis of photoelectron counting and coincidence. From 
a mathematical point of view, renewal processes are especially interesting because their description does not require various assumptions without physical meaning. The only knowledge necessary for their description is the PDF of the intervals between successive points which is effectively an objective of some experiments in statistical optics. The last advantage of renewal PPs is the fact, already noted in [1], that there is a simple relation between the coincidence function and the PDF of the intervals defining the process. This relation will be widely used in our analysis.

On the other hand we cannot say that renewal PPs are effectively DPPs as defined previously and we shall take into account this point in the interpretation of our simulations.

In Sec. IV we show that the set of conditions previously introduced is not sufficient to ensure that a field is classical. For this it is sufficient to show that one can construct an example of renewal PP, which by construction cannot appear for a classical field, but which is such that all the three properties of the set are satisfied. We present the mathematical construction of this PP and the procedure used for its computer simulations. They show clearly that this PP satisfies the set of properties associated with a classical field. In the last two sections we present various examples of PPs satisfying only some of the three properties previously introduced and we discuss the results of some computer simulations corresponding to these examples. One of the most interesting results is to show that it is possible to construct a renewal PP in which there is an antibunching effect while the PDF of the distance between successive points is a decreasing function. This clearly shows that the bunching (antibunching) effect cannot in general be detected by considering only the lifetime of order one of the photoelectrons, as indicated in some studies [8-12].

\section{SHORT REVIEW ON POINT PROCESSES AND THEIR USE IN STATISTICAL OPTICS}

A PP in time is a sequence of random time instants $t_{i}[2,13,14]$. In most of what follows we assume that it is stationary, which means that all the statistical properties are invariant under any change of the origin of time. A $\mathrm{PP}$ is completely defined by the sequence $X_{k}=t_{k}-t_{k-1}$ of distances between successive points called lifetimes of order one. These quantities are positive random variables (RV).

The lifetime of order $n$ is the time between any point $t_{i}$ of the point process and the $n$th point $t_{i+n}$ after $t_{i}$. Let $F_{n}(x)$ and $f_{n}(x)$ be the distribution function (DF) and the probability density function (PDF), when it exists, of the lifetime of order $n$, respectively. These functions appear in the description of various properties of the point process.

Consider first the coincidence analysis. A coincidence event appears when two nonoverlapping small intervals $\left[\theta_{1}, \theta_{1}+\right.$ $\left.d \theta_{1}\right]$ and $\left[\theta_{2}, \theta_{2}+d \theta_{2}\right], \theta_{1}<\theta_{2}$, contain at least one point of the $\mathrm{PP}$, regardless of the number of points of the PP appearing in the interval $\left[\theta_{1}+d \theta_{1}, \theta_{2}\right]$. The calculation of the probability of this event introduces the coincidence function $c(x)$ discussed in Sec. IIA of [1]. It contains all the second-order properties of the PP but also describes the bunching or antibunching effect which was analyzed in various papers (see for example [4]).

For large values of $x$ the coincidence function tends to $\lambda^{2}$, where $\lambda$ is the density of the PP. For a Poisson PP we have $c(x)=\lambda^{2}$ and there is a bunching effect when $c(x)>\lambda^{2}$. For classical fields $c(x)$ belongs to the class of covariance functions, which especially implies that it is maximum for $x=0$. This means that for such fields there is always a bunching effect. One of the purposes of this paper is on the contrary to construct statistical models of PPs in which antibunching appears without invoking quantum properties.

The coincidence function of a stationary PP is related to the PDFs $f_{n}(x)$ of the lifetimes of order $n$ by the relation,

$$
c(x)=\lambda \sum_{n=1}^{\infty} f_{n}(|x|),
$$

also used in [1]. This relation is used for the measurements of $c(x)$ [15] with the series replaced by a sum of a finite number of terms. We shall present various results of such measurements in what follows.

In some experiments the bunching effect is analyzed with the use of $f_{1}(x)$ instead of $c(x)$ (see for example [4]). This is due to the fact that in the case of DS Poisson processes, which means in the case of classical fields, the coincidence function tends to $f_{1}(x)$ when the density tends to 0 , in such a way that the series (2.1) tends to be limited to its first term only.

Another physical argument invoked to consider that the bunching effect can be detected from the study of the coincidence function as well from that of the PDF $f_{1}(x)$ is the following. The difference between the coincidence event defined earlier and the event used to calculate the lifetime of order one is that the number of points of the PP in the interval $\left[\theta_{1}+d \theta_{1}, \theta_{2}\right]$ is zero for the latter while it can take an arbitrary value for the first. But when the density of the PP (i.e., the mean number of points by unit of time) is very small, it can be considered that the probability that this interval contains at least one point is very small, which leads one to conclude that $c(x)$ is proportional to $f_{1}(x)$. We shall see that this argument, correct for DS Poisson processes, is not general and we shall present examples where it cannot be used.

It is, however, interesting to note that the PDF $f_{1}(x)$ can be used as a test in the distinction between classical and nonclassical fields. Indeed, one can show that for a DS Poisson process this PDF is maximum for $x=0$, in such a way that a PP in which this property is not satisfied is certainly nonclassical. The converse property will also be analyzed in the following.

For simplicity of the discussion we shall use the expression conditional bunching effect when it is described only by the use of $f_{1}(x)$, the term conditional coming from the fact that the interval $\left[\theta_{1}+d \theta_{1}, \theta_{2}\right]$ does not contain a point of the PP. One of the conclusions of the following discussion is that a PP can simultaneously exhibit a conditional bunching effect and a standard antibunching effect.

The coincidence function discussed here can be called of second order because it is a coincidence at two distinct time intervals. But it is possible to use coincidence in an arbitrary number $n$ of distinct intervals which introduces coincidence functions of order $n$. The calculation of these functions from the density matrix of the field is the main subject of the paper [17].

Consider now the counting analysis. It is related with the study of the statistical properties of the number of points of the PP registered in some interval $[\theta, \theta+T]$. In the stationary 
case these properties do not depend on $\theta$. These properties are evidently quite different depending on whether or not the time $\theta$ where the observation interval is open is chosen as a point of the PP or not. In the first case we talk about triggered counting and in the latter of relaxed counting. Most of the experimental results published on photon counting are in general related to relaxed counting, and it is also the case in what follows except when it is otherwise specified.

It is clear that there exists a relation between counting and time-interval properties. Let $O$ be an arbitrary point of the PP chosen at the origin of time axis and anterior to $\theta$. Let $S_{n}$ be the RV $X_{1}+X_{2}+?+X_{n}$ which is the distance between $O$ and the $n$th point of the PP posterior to $O$. The probability $p_{m n}(\theta, T)$ that there are $m$ points in the interval $[0, \theta]$ and $n$ points in the interval $[\theta, \theta+T]$ can be expressed in terms of the sums $S_{n}$ by

$$
\begin{aligned}
p_{m n}(\theta, T)= & \mathrm{P}\left[\left(S_{m} \leqslant \theta\right) \cap\left(S_{m+1}>\theta\right) \cap\left(S_{m+n} \leqslant \theta+T\right)\right. \\
& \left.\cap\left(S_{m+n+1}>\theta+T\right)\right] .
\end{aligned}
$$

Its calculation in closed form from the statistical properties of the intervals $X_{i}$ can be very tedious and in some cases the only way to obtain this probability is to use computer simulations described below.

The probability $p_{n}(\theta, T)$ of relaxed counting in the interval $[\theta, \theta+T]$ is of course $p_{n}(\theta, T)=\sum_{m=0}^{\infty} p_{m n}(\theta, T)$ and under general ergodic conditions there is a limit when $\theta$ tends to infinity which is the relaxed counting probability $p_{n}(T)$.

There are several experimental results concerning photon counting in statistical optics. A review of the most significant can be found in [4].

In reality, the probabilities $p_{n}(T)$ are not of a great interest as a test of the classical or nonclassical nature of an optical field. But they can be used to calculate some mean values of the number of points in an interval of duration $T$ and in particular it is shown in [1] [see Eq. (2.16)] that the variance $\sigma_{N}^{2}$ of this number satisfies $\sigma_{N}^{2}=\lambda T+g(T)$, where $\lambda$ is the density of the PP and $g(T)$ a function depending on the second-order properties of the PP. In particular, $g(T)=0$ for a Poisson PP and $g(T)>0$ for a DS Poisson PP. This implies that if there are values of $T$ such that as $g(T)<0$, we can conclude that the field is nonclassical. The reverse question, however, appears immediately: does the fact that $g(T)>0$ for any $T$ imply that the field is classical. We shall analyze this question below.

Note at the end of this section that, since the properties of PPs analyzed in this paper are in relation with classical and nonclassical optical fields and since classical fields introduce necessarily DS Poisson processes, we shall use the expression classical PPs for such PPs. Conversely, all the PPs which are not DS Poisson are called nonclassical PPs.

We can summarize the previous discussion by indicating the three most important properties of a classical PP.

Property A. A classical PP presents a bunching effect, which means that its coincidence function $c(x)$ is maximum for $x=0$.

Property $B$. The PDF $f_{1}(x)$ of a classical PP is maximum for $x=0$. This means that a classical PP exhibits conditional bunching effect.

Property $C$. The variance of the number or points of a classical PP in relaxed counting satisfies $\sigma_{N}^{2}>\lambda T$.
Let us now discuss more precisely the concept of DPP introduced in Sec. I. As indicated previously the statistical properties of an optical field are completely described by its density matrix $\rho$. From this density it is possible to calculate the so-called quantum $n$ th-order correlation functions defined in [16] and called earlier higher-order coincidence functions. They are used for the calculations of coincidence phenomena in photodetection phenomena of the optical field defined by $\rho$ as indicated in [17].

Furthermore it is shown in [18] that the statistical properties of a PP can be completely mathematically defined from the set of its coincidence functions. Combining these two properties we can say that a DPP is defined by a density matrix $\rho$. Unfortunately, the complete calculations are extremely complicated and can be completed only in a small number of cases.

The inverse problem is still more complicated. It consists in finding the density matrix $\rho$ of a DPP defined by the set of all its coincidence functions. The question of knowing whether or not there are mathematical PPs that are not DPPs, which means that the inverse problem is without solution remains an open problem.

\section{ANALYSIS AND SIMULATION OF SOME RENEWAL PROCESSES}

Renewal processes are characterized by the fact that the time intervals $X_{k}$ between successive points of the PP are independent and identically distributed (IID) random variables.

Unfortunately, the fact that renewal PPs are DPPs remains an open conjecture and its proof seems a very difficult task. We shall take this fact into consideration in our following discussion. However, if renewal PPs were DPPs, the corresponding optical field would certainly be not classical, because a doubly stochastic Poisson process cannot introduce independent intervals except in the case when it is a pure Poisson process.

A renewal PP is entirely defined by the PDF $f_{1}(x)=f(x)$ of the time intervals $X_{k}$. This simplifies significantly the use of (2.1). Indeed it results from the independence assumption that the generating function $G_{n}(s)$ of the $\operatorname{PDF} f_{n}(x)$ is simply $G^{n}(s)$, where $G(s)$ is the generating function of $f(x)$. As $|G(s)|<1$, the monolateral Laplace transform of $c(x)$ becomes

$$
C(s)=\lambda \sum_{n=1}^{\infty} G^{n}(s)=\lambda \frac{G(s)}{1-G(s)} .
$$

Note that, since $c(x)$ is an even function of $x$, its complete Laplace transform is $\hat{C}(s)=C(s)+C(-s)$. The Fourier transform of $c(x)$ is, of course, $\hat{C}(i \omega)$. It is well known that the density $\lambda$ of a renewal process is the inverse of the mean value $m_{X}$ of the time between successive points. As the generating function $G(s)$ satisfies $G(s)=\mathrm{E}[\exp (-s X)]=1-m_{X} s+o\left(s^{2}\right)$, the application of the expression $\lim _{x \rightarrow \infty} c(x)=\lim _{s \rightarrow 0} s C(s)$ yields the property already indicated and that $c(x)$ tends to $\lambda^{2}$ when $x$ tends to infinity.

Expression (3.1) implies that the coincidence function of a renewal PP must satisfy certain constraints. The most evident 
comes from the fact that the generating function of the lifetime cannot be an arbitrary function because its inverse Laplace transform must be non-negative and equal to zero for $x<0$, since it is the PDF of a positive RV. It is, however, difficult to determine the exact consequences of these conditions on the coincidence function. We shall discuss the problem below with some specific examples.

The properties of the coincidence function suggest an important problem in our discussion: Is it possible to associate a renewal PP with a given coincidence function? For this purpose it is necessary to make an inversion of (3.1) which yields

$$
G(s)=\frac{C(s)}{\lambda+C(s)} .
$$

But in order to complete the problem it is necessary to verify that the function $G(s)$ obtained from this equation is effectively the generating function of a positive RV. There is no general solution to this problem but we shall discuss it with the help of some examples.

The simplest one is when $c(x)=1$, which is the coincidence function of a Poisson process of density $\lambda=1$. It results from (3.2) that $G(s)=1 /(s+1)$ which is the generating function of an exponential distribution of unit mean value. The corresponding renewal process is a Poisson process of unit density, and, as indicated earlier, its coincidence function is constant.

Let us now verify this in a computer simulation similar to those presented in [1]. A Poisson process is a renewal process in which the $\operatorname{PDF} f_{1}(x)$ is an exponential distribution. The first step is then to generate a large number $N$ of samples $X_{k}$ of IID random variables with a unit exponential distribution. This is easily done by using the transformation $X_{k}=-\log \left(1-U_{k}\right)$, where $U_{k}$ is a sequence of IID random variables uniformly distributed in $[0,1]$. The numerical simulation is done by measuring distinct values of the PDFs $f_{k}(x)$ appearing in (2.1) by using standard procedures from normalized histograms.

The results appear in Fig. 1. The points indicate the results of the PDF measurements of the first four PDFs $f_{k}(x)$.

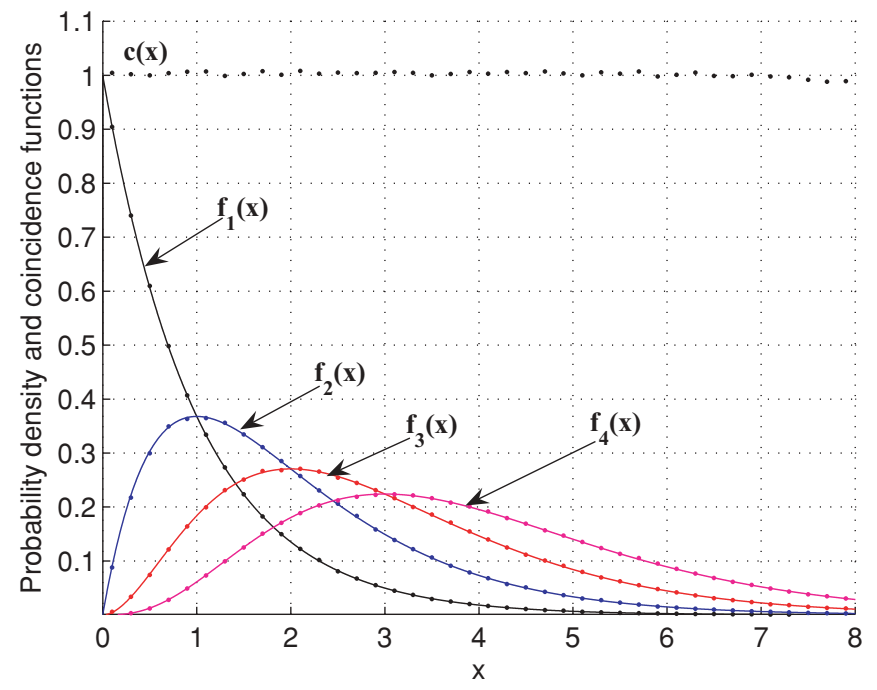

FIG. 1. (Color online) Coincidence function $c(x)$ and PDFs $f_{k}(x), 1 \leqslant k \leqslant 4$, for a Poisson process of density $\lambda=1$. Points, computer experiment; continuous curves, theory.
The continuous curves are the analytical values of these functions equal in the case of a Poisson process of unit density to $f_{k}(x)=x^{k-1} \exp (-x) /(k-1)$ !. We observe an excellent agreement between analytical and simulation results. For the measurement of $c(x)$ we use (2.1) in which only 15 terms are taken into account. These terms are PDFs measured as those presented above. We observe that for values of $x$ smaller than 8 the coincidence function measured is practically equal to 1 , which is the value expected for a Poisson process of unit density. This result can be considered as a test of the validity of the procedure of measurement of the coincidence function which will be used many times further below.

\section{NONSUFFICIENCY OF THE SET OF PROPERTIES A, B, AND C}

In this section we shall answer the question stated in Sec. I and show that it is possible to construct a renewal PP, which is by definition nonclassical, but which exhibits the same bunching effect as a classical PP. In reality the result is much stronger. We shall show that a nonclassical PP can satisfy simultaneously the three properties A, B, and C, which are sometimes considered as characteristic of a classical PP.

The coincidence function with a decreasing exponential shape is the most common example introducing a bunching effect. It appears in many physical situations, as, for example, in the case of thermal fields generated by a first-order Langevin equation (see, e.g., [19]). Consider then the function $c(x)=$ $\lambda^{2}[1+k \exp (-a|x|)], k>0, a>0$. The problem is then to find a renewal PP in which $c(x)$ is its coincidence function. Applying (3.2) yields

$$
G(s)=\lambda \frac{\alpha s+a}{s^{2}+(a+\alpha \lambda) s+a \lambda},
$$

where $\alpha=1+k$. We have $G(0)=1$, as for any generating function, and the only point to verify is whether its Laplace inverse transform is positive in order to be a PDF. This results directly from the fact that the roots of the denominator are real

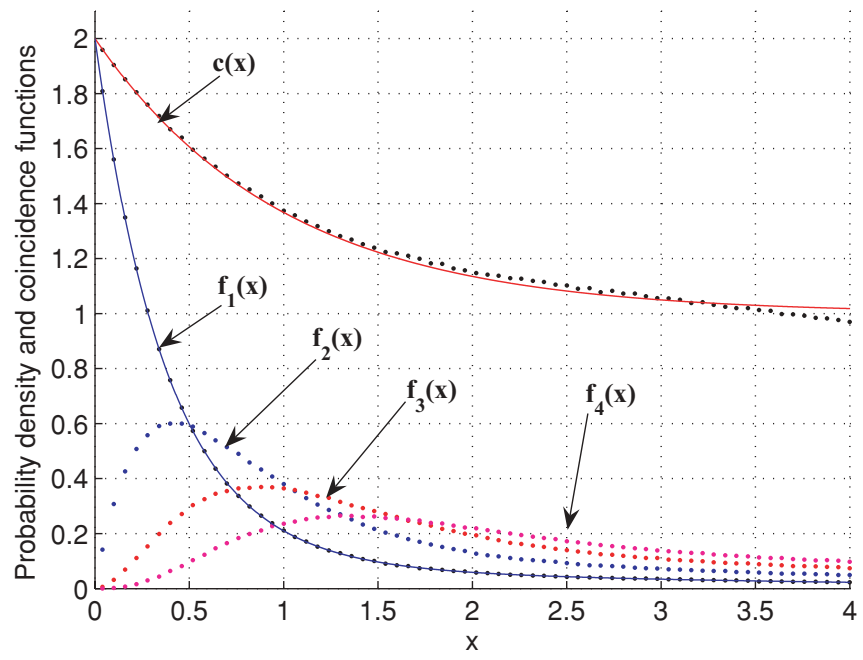

FIG. 2. (Color online) Coincidence function $c(x)$ and PDFs $f_{k}(x), 1 \leqslant k \leqslant 4$, for the renewal process defined by (4.2). Points, computer experiment; continuous curves, theory. 
TABLE I. First 10 values of counting probabilities.

\begin{tabular}{|c|c|c|c|c|c|c|c|c|c|c|c|}
\hline & $T$ & $p_{0}$ & $p_{1}$ & $p_{2}$ & $p_{3}$ & $p_{4}$ & $p_{5}$ & $p_{6}$ & $p_{7}$ & $p_{8}$ & $p_{9}$ \\
\hline 1 & 1 & 0.3679 & 0.3679 & 0.1839 & 0.0613 & 0.0153 & 0.0031 & 0.0005 & 0.0001 & 0.0000 & 0.0000 \\
\hline 2 & 1 & 0.3677 & 0.3681 & 0.1839 & 0.0613 & 0.0153 & 0.0031 & 0.0005 & 0.0001 & 0.0000 & 0.0000 \\
\hline 3 & 1 & 0.5141 & 0.1996 & 0.1453 & 0.0830 & 0.0375 & 0.0142 & 0.0046 & 0.0013 & 0.0003 & 0.0001 \\
\hline 4 & 2 & 0.1353 & 0.2707 & 0.2707 & 0.1804 & 0.0902 & 0.0361 & 0.0120 & 0.0034 & 0.0009 & 0.0002 \\
\hline 5 & 2 & 0.1355 & 0.2708 & 0.2707 & 0.1801 & 0.0902 & 0.0360 & 0.0120 & 0.0035 & 0.0009 & 0.0000 \\
\hline 6 & 2 & 0.3374 & 0.1596 & 0.1502 & 0.1267 & 0.0953 & 0.0618 & 0.0359 & 0.0185 & 0.0087 & 0.0036 \\
\hline
\end{tabular}

and negative, which implies that the inverse Laplace transform is a sum of two causal and decreasing exponential functions.

Let us present an example of such an identification problem. Suppose that $\lambda=1$ and $k=1$, which yields $\alpha=2$. From simple algebra we deduce that the probability density function corresponding to (4.1) is

$$
f(x)=u(x)\left[p_{1} a_{1} \exp \left(-a_{1} x\right)+p_{2} a_{2} \exp \left(-a_{2} x\right)\right],
$$

where $u(x)$ is the unit step function and $p_{1}=(b+2) / a_{1} b$, $p_{2}=1-p_{1}, a_{1}=(a+b+2) / 2, a_{2}=(a-b+2) / 2$, and $b=\sqrt{a^{2}+4}$. It is clear that the corresponding mean value is equal to 1 , because of the assumption $\lambda=1$.

We shall now verify these results in a simulation. There is a simple way to generate a sequence of IID random variables $X_{k}$ with the PDF (4.2). Let $\bar{U}_{k}$ be a set of IID random variables uniformly distributed in $[0,1]$. It is clear that the random variables $U_{k}$ defined by $U_{k}=\left(-1 / a_{1}\right) \ln (1-\bar{U} k)$ are IID and their PDF is $a_{1} \exp \left(-a_{1} x\right)$. By using the same procedure we can generate a second sequence of IID exponential random variables $V_{k}$, independent of the $U_{k}$. Let finally $B_{k}$ be a sequence of Bernoulli random variables, independent of $U_{k}$ and $V_{k}$, taking the values 0 or 1 with $\operatorname{pr}\left(B_{k}=1\right)=p_{1}$. All these random variables can easily be obtained in a computer simulation. Consider now the random variables defined by

$$
X_{k}=B_{k} U_{k}+\left(1-B_{k}\right) V_{k} .
$$

It is clear that these random variables are IID and that their PDF is precisely (4.2). The simulation procedure is the same as in Fig. 1, but in this case the analytical expressions of the PDF $f_{k}(x)$ do not have a simple form, except for $k=1$ where it is given by (4.2). The results for $a=1$ appear in Fig. 2 . We observe that the coincidence function is correctly estimated and the simulated points are located on the continuous curve defined by $1+\exp (-x)$. The same is valid for $f_{1}(x)$ with the continuous curve corresponding to (4.2). At this step we see that properties A and B are satisfied, even if the PP analyzed is nonclassical because it is a renewal process.

TABLE II. Mean values and variances of the number of points.

\begin{tabular}{lcc}
\hline \hline$T$ & Mean value & Variance \\
\hline $1 / 2$ & 0.5002 & 0.7120 \\
1 & 1.0001 & 1.7391 \\
2 & 2.0000 & 4.2534 \\
3 & 2.9997 & 7.1057 \\
\hline
\end{tabular}

It remains to consider counting properties to verify property C. In [20] we have presented a procedure which allows us to deduce the probabilities $p_{n}$ of relaxed counting defined earlier from the sequence of lifetimes $X_{k}$. Data analysis using this method is displayed in the following. In Table I we present measurements of the probabilities $p_{n}$.

All these results correspond to PPs with density $\lambda=1$. The first three lines are obtained with $T=1$, and the others with $T=2$. Lines 1 and 4 display theoretical results for a stationary Poisson PP. Lines 2 and 5 correspond to results of simulations obtained with the same PPs. We observe that results of lines 1 and 2 or lines 4 and 5 are very similar, which can be considered as a test of the correct behavior of the method. Results of lines 3 and 6 correspond to the PP studied in this section and defined by (4.3). The interpretation, however, of these results in terms of distinction between classical and nonclassical $\mathrm{PP}$ is rather complicated. For this it is more efficient to use the test of the variance appearing in property $\mathrm{C}$. By using experimental values of the probabilities $p_{n}$ presented, for example, in Table I we can calculate the corresponding mean value and variance of the number of points registered in an interval of duration $T$. The results appear in Table II. This table requires two main comments. At first we verified that the mean value is effectively given by the expression $\lambda T$ because, as said earlier, the density of the PP generated by (4.3) is $\lambda=1$. This was also clear on the asymptotic value of $c(x)$ appearing in Fig. 2. Secondly, the comparison

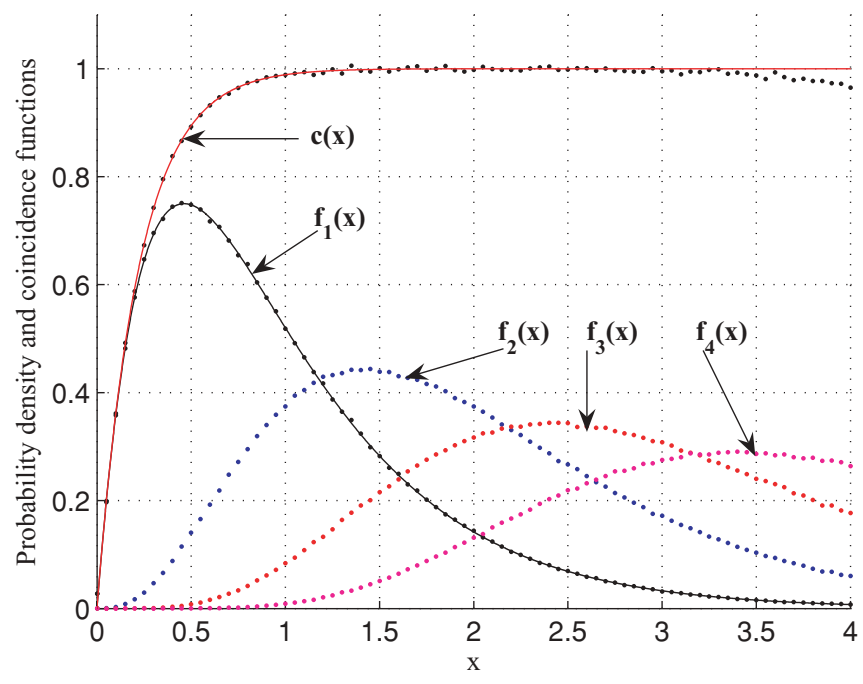

FIG. 3. (Color online) Coincidence function $c(x)$ and PDFs $f_{k}(x)$, $1 \leqslant k \leqslant 4$, for the renewal process defined by (5.2) with $a=9 / 2$. Points, computer experiment; continuous curves, theory. 
TABLE III. First 10 values of counting probabilities.

\begin{tabular}{ccccccccc}
\hline \hline$p_{0}$ & $p_{1}$ & $p_{2}$ & $p_{3}$ & $p_{4}$ & $p_{5}$ & $p_{6}$ & $p_{7}$ & $p_{8}$ \\
\hline 0.2812 & 0.4798 & 0.2007 & 0.0348 & 0.0033 & 0.0002 & 0.0000 & 0 & 0 \\
\hline \hline
\end{tabular}

between mean and variance means that property $\mathrm{C}$ is satisfied.

In conclusion, we see that the PP studied in this section which is nonclassical because it is a renewal process satisfies all the three main properties of a classical PP. This clearly shows that these properties are not sufficient to characterize the classical character of a PP.

This conclusion is perfectly correct when it is expressed in terms of point processes, as is the case. Its formulation in terms of optical fields would require the proof of the conjecture that renewal processes are DPPs, as indicated earlier.

\section{EXAMPLE OF RENEWAL PROCESSES IN WHICH THE THREE CONDITIONS A, B, AND C ARE NOT SATISFIED}

The simplest example of coincidence function introducing the antibunching effect is the increasing exponential function such as $c(x)=\lambda^{2}[1-\exp (-a|x|)]$. We shall first see that it is not always possible to associate a renewal process to this function. Indeed (3.2) yields

$$
G(s)=\lambda \frac{a}{s^{2}+a s+a \lambda},
$$

and the poles are real and negative if $a>4 \lambda$. Otherwise these poles are complex and the inverse Laplace transform of $G(s)$ cannot be positive. Thus, if $a<4 \lambda$ there is no renewal process having $c(x)$ as its coincidence function. Suppose now that $\lambda=1$ and $a>4$. It is easy to find that the PDF associated with (5.1) is

$$
f(x)=2(a / b) \exp (-a x / 2) \sinh (b x / 2),
$$

with $b=\sqrt{a^{2}-4 a}$. Its mean value is 1 , because of the condition $\lambda=1$.

Because of the difference of two exponential functions appearing in the term $\sinh (b x / 2)$ of $(5.2)$, it is not possible to use a procedure like (4.3) for a simulation of random variables $X_{k}$ that are IID and defined by the probability density function (5.2). Fortunately, for some particular values of $a$ a direct inversion of the DF is possible, in such a way that the random variables $X_{k}$ can be generated from a sequence of random variables $U_{k}$ that are IID and uniformly distributed. This appears, for example, when $a=9 / 2$. In

TABLE IV. Mean values and variances of the number of points.

\begin{tabular}{lcc}
\hline \hline$T$ & Mean value & Variance \\
\hline $1 / 2$ & 0.4995 & 0.3655 \\
1 & 0.9998 & 0.6541 \\
2 & 1.9995 & 1.2126 \\
3 & 2.9996 & 1.7616 \\
\hline \hline
\end{tabular}

this case a simple calculation shows that the transformation $X_{k}=-(2 / 3) \log \left(1-\sqrt{U_{k}}\right)$ applied to random variables $U_{k}$ that are IID and uniformly distributed in $[0,1]$ generates a set of random variables that are IID and with the PDF (5.2). Furthermore, when these random variables are the lifetime of order one of a PP, its coincidence function is $1-\exp (-a x)$.

Results of simulations appear in Fig. 3. The numerical values of the coincidence function corresponds exactly to the theoretical values, and this is also true for the PDF $f_{1}(x)=f(x)$ where $f(x)$ is given by (5.2). On the other hand there is no simple expression of the other PDFs $f_{n}(x)$ for $n>1$. Note that because of the value of $a$, the asymptotic value 1 is reached faster than that in the previous figures, and this allows for the estimation of $c(x)$ with a smaller number of terms in (2.1). The results of Fig. 3 are obtained with only seven terms in this sum and in the interval $0<x<3$ this is sufficient to obtain a good approximation to the coincidence function. Note also that for small values of $x$ the coincidence function is well approximated by using only the PDF of the lifetime of order one.

It is important to note that the coincidence function $c(x)$ appearing in Fig. 3 is not a covariance function because it has a minimum for $x=0$. It exhibits a clear antibunching effect. We note that the increase of $c(x)$ for small values of $x$ comes from the facts that the $\operatorname{PDF} f_{1}(x)=f(x)$ of the random variables $X_{k}$ is zero for $x=0$ and that the other PDFs $f_{k}(x)$ are almost 0 for small values of $x$.

It is clear in Fig. 3 that properties A and B are not satisfied. It remains then to study the counting properties. In Table III we present results similar to those of Table I but for the renewal process, some properties of which appear in Fig. 3. This PP

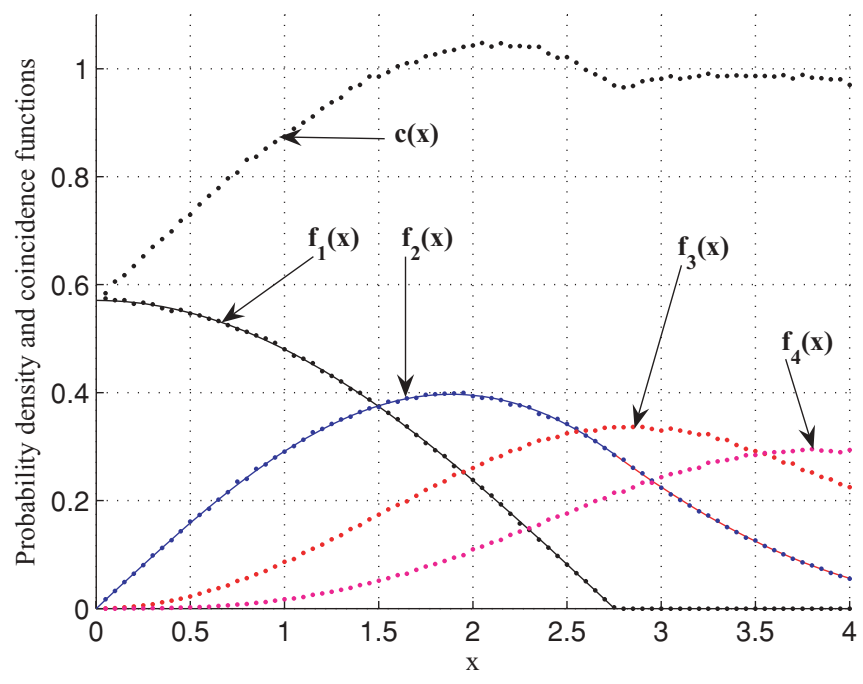

FIG. 4. (Color online) Coincidence function $c(x)$ and PDFs $f_{k}(x)$, $1 \leqslant k \leqslant 4$, for the renewal process defined in Sec. VI. Points, computer experiment; continuous curves, theory. 
TABLE V. First 10 values of counting probabilities.

\begin{tabular}{lccccccccc}
\hline \hline$p_{0}$ & $p_{1}$ & $p_{2}$ & $p_{3}$ & $p_{4}$ & $p_{5}$ & $p_{6}$ & $p_{7}$ & $p_{8}$ \\
\hline 0.2776 & 0.4973 & 0.1800 & 0.0382 & 0.0061 & 0.0007 & 0.0001 & 0.0000 & 0.0000 & 0 \\
\hline \hline
\end{tabular}

is defined by the PDF defined by (5.2) with $a=9 / 2$. Its density is $\lambda=1$, as it appears on the asymptotic value of its coincidence function, and $T=1$. We observe that there are only six nonzero values of the counting probabilities $p_{n}$ and this is an important difference with the case of line 3 of Table I corresponding to the same values of the parameters $\lambda$ and $T$.

On Table IV we present results similar to those presented in Table II. As in this table the mean values correspond to the theoretical value $\lambda T$. On the other hand for all the values of $T$ considered, the variance $\sigma^{2}$ is smaller that the mean value, which shows that property $\mathrm{C}$ is not verified.

This clearly shows that the PP considered in this section does not satisfy each of the three properties necessary for ensuring that a PP is classical.

\section{EXAMPLE OF RENEWAL PROCESSES WHERE CONDITION B IS SATISFIED AND CONDITIONS A AND C ARE NOT SATISFIED}

Consider the renewal process defined by the PDF $f(x)=$ $f_{1}(x)=(\pi / 2 a) \cos (\pi x / 2 a)$ for $0 \leqslant x \leqslant a$ and $f(x)=0$ otherwise. If $a=\pi /(\pi-2)$ its mean value is one, which is also the value of the density $\lambda$ of the PP. Since the calculation of $c(x)$ from (3.1) is almost impossible, a simulation is the only way to obtain this function. For this it is necessary to generate the random variables $X_{k}$, which is easy from uniformly distributed random variables with the transformation $X_{k}=$ $(2 a / \pi) \arcsin \left(U_{k}\right)$. The results appear in Fig. 4. The calculation of the convolution $f_{2}(x)$ is still rather simple. The simulated points of $f_{1}(x)$ and $f_{2}(x)$ are located on the calculated curves. There is no simple expression for the other convolutions that yield the functions $f_{k}(x)$ for $k>2$. The fact that $c(x)$ is increasing while $f_{1}(x)$ is decreasing comes from the fact that all the other probability density functions are increasing, while $f_{1}(x)$ is almost constant for small values of $x$. There are various other examples of the same situation. This appears especially when the PDF $f_{1}(x)$ is decreasing but with a derivative $f_{1}^{\prime}(x)$ equal to zero for $x=0$. In this case the increase of the other PDFs $f_{n}(x)$ for small values of $x$ is sufficient to ensure that the coincidence function $c(x)$ increases in the neighborhood of the origin, which implies antibunching effect.

TABLE VI. Mean values and variances of the number of points.

\begin{tabular}{lcc}
\hline \hline$T$ & Mean value & Variance \\
\hline $1 / 2$ & 0.5002 & 0.4068 \\
1 & 1.0003 & 0.6781 \\
2 & 2.0006 & 1.0821 \\
3 & 3.0002 & 1.5218 \\
\hline
\end{tabular}

This result is important because it shows clearly that in this case it is impossible to approximate the coincidence function by using only $f_{1}(x)$ as this appears for a classical PP. The coincidence function shows clearly that there is an antibunching effect while considering the PDF $f_{1}(x)$ shows a conditional bunching effect.

The first values of the counting probabilities appear in Table V. Furthermore, Table VI obtained with the same values of $T$ as in Tables II and IV shows clearly that the variance is smaller than the mean value, which means that condition $\mathrm{C}$ is not satisfied.

Finally, it is interesting to note that the converse example is not possible. Indeed, if the PDF $f_{1}(x)$ increases for small values of $x$, as, for example, in the case appearing in Fig. 3, then the coincidence function $c(x)$ necessarily increases as well, which means that an antibunching effect occurs. Indeed, it is easy to verify that for a renewal PP the PDFs $f_{n}(x)$ satisfy $f_{n}(0)=0$, which implies that they are increasing for small values of $x$. Since, according to $(2.1), c(x)$ is a sum of increasing functions, it also increases. This can be summarized in the following proposition: In the case of renewal PPs, a conditional antibunching effect implies an ordinary antibunching effect.

\section{CONCLUSION}

Let us highlight the two main results of this paper. The first one is that the interpretation of results of experiments of statistical optics requires a great care when the aim is to decide whether or not an optical field is classical. Indeed, a classical field exhibits three main properties: (A) a bunching effect; (B) a bunching conditional effect; (C) a variance of the number of events recorded in any interval greater that its mean value. But these three properties can also appear with nonclassical PPs and we have presented examples of this situation. It results from this fact that they are insufficient to yield an answer to the question indicated previously.

The second one is that it is important to avoid any confusion between a bunching effect detected by using the coincidence function and a conditional bunching effect detected by using the PDF of the distance between successive points of the PP. The argument that these two distinct effects are identical for PPs with very low density is mathematically incorrect and we have presented specific examples illustrating this fact.

\section{ACKNOWLEDGMENTS}

The Laboratoire des Signaux et Systèmes is a joint laboratory (UMR 8506) of Centre National de la Recherche Scientifique and École Supérieure d'Électricité and associated with the Université de Paris-Sud, Orsay, France. 
[1] B. Picinbono and C. Bendjaballah, Phys. Rev. A 71, 013812 (2005).

[2] D. L. Snyder, in Random Point Processes (Wiley, New York, 1975), Chapter 6.

[3] H. J. Kimble, M. Dagenais, and L. Mandel, Phys. Rev. Lett. 39, 691 (1977).

[4] L. Mandel and E. Wolf, Optical Coherence and Quantum Optics (Cambridge University Press, New York, 1995), Chapter 14.

[5] T. Jeltes, J. M. McNamara, W. Hogervorst, W. Vassen, V. Krachmalnicoff, M. Schellekens, A. Perrin, H. Chang, D. Boiron, A. Aspect, and C. I. Westbrook, Nature (London) 445, 402 (2007).

[6] C. Bendjaballah and M. Pourmir, Phys. Rev. A 79, 053826 (2009).

[7] C. Bendjaballah, Phys. Rev. A 73, 053816 (2006).

[8] R. Brouri, A. Beveratos, J.-P. Poizat, and P. Grangier, Opt. Lett. 25, 1294 (2000).

[9] A. Beveratos, R. Brouri, T. Gacoin, A. Villing, J.-P. Poizat, and P. Grangier, Phys. Rev. A 64, 061802(R) (2001).

[10] Y. Takayama et al., J. Synchrotron Radiat. 10, 303 (2003).

[11] Y. H. Kim, Phys. Lett. A 315, 352 (2003).
[12] M. Halder, S. Tanzilli, H. de Riedmatten, A. Beveratos, H. Zbinden, and N. Gisin, Phys. Rev. A 71, 042335 (2005).

[13] D. R. Cox and V. Isham, in Point Processes (Chapman and Hall, London, 1980), Chapters 1 and 2.

[14] D. J. Daley and D. Vere-Jones, An Introduction to the Theory of Point Processes, Elementary Theory and Methods, Vol. I. (Springer, New York, 2003).

[15] B. Picinbono, IEEE Trans. Instrum. Meas. 57, 548 (2008).

[16] R. J. Glauber, in Quantum Optics and Electronics (Les Houches 1964), edited by C. de Witt, A. Blandin, and C. Cohen-Tannoudji (Gordon and Breach Science Publishers, New York, 1965), p. 88.

[17] P. L. Kelley and W. H. Kleiner, Phys. Rev. 136, A316 (1964).

[18] O. Macchi, IEEE Trans. Inf. Theory 17, 2 (1971).

[19] C. W. Gardiner, in Handbook of Stochastic Methods for Physics, Chemistry and the Natural Sciences (Springer, Berlin, 1989), Chapter 1.

[20] B. Picinbono, "Comptages déclenchés et relaxés dans les processus ponctuels," L2S Internal Report [ftp://ftp.lss.supelec.fr/pub/users/picinbono/2. Reports/]. 